\title{
Geleitwort der Bundesministerin für EU und Verfassung
}

Das 100. Jubiläum unserer Verfassung am 1. Oktober 2020 diente den Herausgebern des vorliegenden Tagungsbandes als willkommener Anlass zur Auseinandersetzung mit dem Bundesstaat. Dieses Jubiläum fiel in ein Jahr, das uns mit vielen unerwarteten Herausforderungen konfrontiert hat, sodass es schwer war, innezuhalten, um es entsprechend zu feiern. Die Umstände haben uns aber auch erinnert, nicht nur zurück zu schauen, sondern unsere Verfassung dafür zu würdigen, dass sie - wie vor 100 Jahren - den Rahmen unseres staatlichen Handelns bildet. Gerade Krisen zeigen die Wichtigkeit unserer Institutionen und der Rechtsordnung auf, um Stabilität und Orientierung zu geben. So gab es nur wenige Festakte, dafür aber zahlreiche öffentliche Diskussionen, die das Bewusstsein über die Garantien unserer Verfassung gestärkt haben. Auch mit einem Jahr Abstand bin ich froh, dass die Maßnahmen zur Bewältigung der Corona-Pandemie in Österreich stets auf Grundlage parlamentarischer Entscheidungen getroffen wurden. Damals wie heute trachten wir danach, dass die Freiheitsbeschränkungen so gering und kurz wie möglich gehalten werden, um das Ziel zu erreichen, die Funktionsfähigkeit des Gesundheitssystems aufrecht zu erhalten.

Die Kompetenzverteilung ist ein wesentlicher Eckpfeiler unserer Verfassung. Sie ist Teil der österreichischen Verfassungsidentität. Gesetzgebung und Vollziehung sind zwischen Bund und Ländern aufgeteilt. Insbesondere durch den österreichischen Beitritt zur Europäischen Union wurden viele Zuständigkeiten auf Unionsebene gehoben. Trotz dieser und weiterer Änderungen hält sich das System der Aufteilung von Macht und Verantwortung in weiten Teilen seit 100 Jahren stabil und hat sich dem Grunde nach bewährt. An die Kompetenzverteilung, wie wir sie kennen, haben wir uns gewöhnt. Gerade deshalb sind die Beiträge, die Sie in diesem Band erwarten, so wichtig: Sie zeigen nicht nur die Entwicklung des jetzigen Systems auf, sondern auch, welche Formen sich in anderen Rechtsordnungen gebildet haben und welche Alternativen es aus historischer Sicht gab. Ein solcher Blick über den zeitlichen und örtlichen Tellerrand lohnt immer, gerade auch aus der heutigen Perspektive als Teil der Europäischen Union. Die Ergebnisse erinnern uns auch daran, dass Dinge nicht immer so bleiben müssen, wie sie sind. 
Den Herausgebern, Autorinnen und Autoren dieses Bandes möchte ich zum vorliegenden Tagungsband gratulieren. Zudem danke ich ihnen dafür, dass sie aus Anlass des 100-jährigen Jubiläums unserer Verfassung auch die Kompetenzverteilung, den Kern des bundesstaatlichen Prinzips, in dieser Tiefe würdigen. Den hoffentlich zahlreichen Leserinnen und Lesern wünsche ich eine interessante und inspirierende Lektüre!

Wien, 31. August 2021

Mag. Karoline EDTSTADLER 